\title{
TCEC Cup 4
}

Article

Accepted Version

The TCEC Cup 4 report

Haworth, G. and Hernandez, N. (2020) TCEC Cup 4. ICGA Journal, 41 (4). pp. 259-263. ISSN 1389-6911 doi: https://doi.org/10.3233/ICG-190124 Available at https://centaur.reading.ac.uk/86831/

It is advisable to refer to the publisher's version if you intend to cite from the work. See Guidance on citing.

Published version at: https://content.iospress.com/articles/icga-journal/icg 190124

To link to this article DOI: http://dx.doi.org/10.3233/ICG-190124

Publisher: The International Computer Games Association

All outputs in CentAUR are protected by Intellectual Property Rights law, including copyright law. Copyright and IPR is retained by the creators or other copyright holders. Terms and conditions for use of this material are defined in the End User Agreement.

\section{www.reading.ac.uk/centaur}

\section{CentAUR}

Central Archive at the University of Reading

Reading's research outputs online 


\section{TCEC Cup 4}

Guy Haworth and Nelson Hernandez ${ }^{1}$

Reading, UK and Maryland, USA

The TCEC Cup knockout event was this time held after the host TCEC 16 league event finished rather than before the Superfinal (Haworth and Hernandez, 2019a, 2019b). TCEC Cup 4 began on October $16^{\text {th }} 2019$, in synchrony with the knockout phase of the Rugby World Cup.

Some authors moved away from their TCEC16 engine versions as noted in Tables 1-5. It is not always possible to decrypt version numbers to understand whether the move is forwards, backwards or sideways. Nevertheless, TCEC clearly encourages active development in the engine shed (CPW, 2019). Engine versions and nominal ELOs did not change between rounds. The engine logos are listed in Fig. 1 in seed order.

The 'standard pairing' was again used, with seed $s$ playing seed $2^{6-r}-s+1$ in round $r$ if the wins all go to the higher seed. Thus, seed $s 1$ plays $s 32, s 16, \ldots, s 2$ if all survive long enough. The higher seed is listed first in Table 1. As for TCEC Cup 3 (Haworth and Hernandez, 2019c), the matches - eight games plus any necessary game-pair tiebreaks - were played out only until the result was decided.

The usual 'TCEC opening' team, the second author here and Jeroen Noomen, randomly chose from three books with some regard for frequency over the board. This provides greater variety of play for the rounds up to and including the semi-finals. The final took openings of various lengths from JN's TCEC Superfinal books for seasons 9-15.
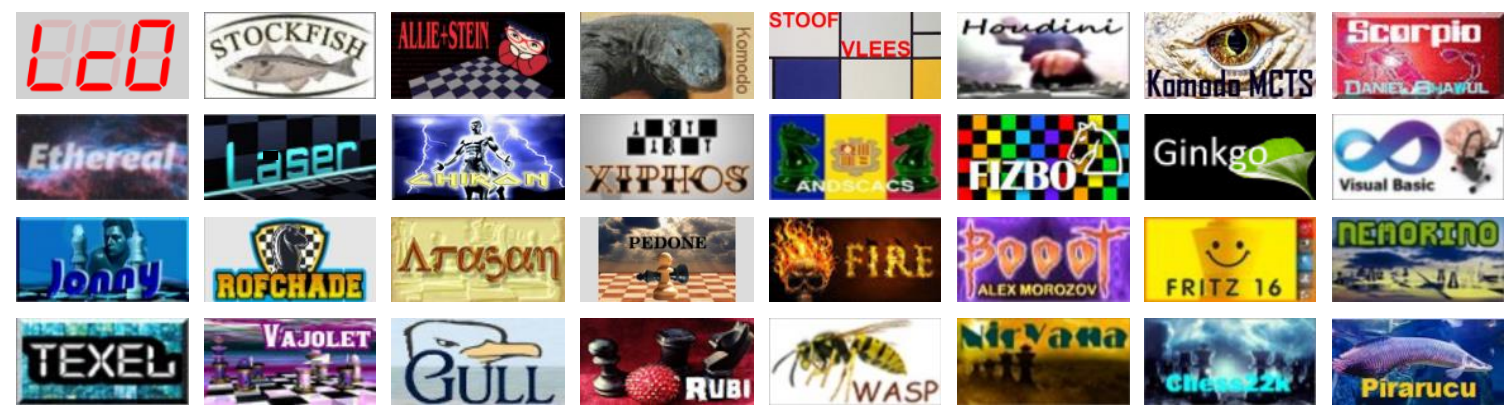

Fig. 1. Logos for TCEC Cup engines in seeded order (LeELA CHESS Zero $\rightarrow$ STOCKFISH $\rightarrow \ldots \rightarrow$ PIRARUCU).

As in previous TCEC Cup events, interest focused on the winner's actual performance ' $\% \mathrm{P}$ ' compared with their expected performance ' $\mathrm{E} \% \mathrm{P}$ ' implied by TCEC ELO difference 'ELO $\Delta$ '. The accuracy of the TCEC ELOs especially for the newer engines, the upgrades to over half the field and the influence of the random openings were again the main uncertainties.

\footnotetext{
${ }^{1}$ Corresponding author: g.haworth@reading.ac.uk
} 
Table 1. TCEC Cup 4: round one results from the winner's perspective. ${ }^{2}$

\begin{tabular}{|c|c|c|c|c|c|c|c|c|c|c|c|c|}
\hline$\alpha \beta$ & $\#$ & 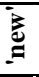 & $\begin{array}{c}\text { Elo } \\
\Delta\end{array}$ & Elo & 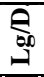 & ర్d & Round 1 Pairings & $\mathbf{E \% P}$ & Round 1 Results & $\# \mathbf{g}$ & $\% \mathbf{P}$ & par.. \\
\hline $\begin{array}{l}\text { Lc } \\
\text { pi }\end{array}$ & 01 & $\begin{array}{l}\checkmark \\
\times\end{array}$ & 384 & $\begin{array}{l}3901 \\
3517\end{array}$ & $\begin{array}{l}\mathrm{P} \\
\mathrm{Q}\end{array}$ & $\begin{array}{l}01 \\
32\end{array}$ & $\begin{array}{l}\text { LCZero v0.22.0-nT2 } \\
\text { pirarucu 3.0.7 }\end{array}$ & 90.12 & Leela Chess Zero, 5-2: 1=1===1 & 7 & 71.43 & --- \\
\hline $\begin{array}{l}\mathrm{Cb} \\
\mathrm{Jo}\end{array}$ & 02 & $\begin{array}{l}x \\
\times\end{array}$ & 38 & $\begin{array}{l}3660 \\
3622\end{array}$ & $\begin{array}{l}1 \\
1\end{array}$ & $\begin{array}{l}16 \\
17\end{array}$ & $\begin{array}{c}\text { ChessBrainVB } 3.74 \\
\text { Jonny } 8.1\end{array}$ & 55.45 & $\begin{array}{l}\text { ChessBrainVB, 51/2-41/2: } \\
\quad=01001=1,1=\end{array}$ & 10 & 55.00 & $=$ \\
\hline $\begin{array}{l}\mathrm{Sn} \\
\mathrm{Te}\end{array}$ & 03 & $\begin{array}{l}\checkmark \\
\times\end{array}$ & 76 & $\begin{array}{l}3707 \\
3631\end{array}$ & $\begin{array}{l}1 \\
2\end{array}$ & $\begin{array}{l}08 \\
25\end{array}$ & $\begin{array}{c}\text { ScorpioNN v3.0.3-n_maddex_INT8 } \\
\text { Texel } 1.08 \mathrm{a} 13\end{array}$ & 60.77 & ScorpioNN, 5-0: 11111 & 5 & 100.00 & H+r \\
\hline $\begin{array}{l}\mathrm{Et} \\
\mathrm{Ne}\end{array}$ & 04 & $\begin{array}{l}\checkmark \\
\checkmark\end{array}$ & 119 & $\begin{array}{l}3746 \\
3627\end{array}$ & $\begin{array}{l}1 \\
2\end{array}$ & $\begin{array}{l}09 \\
24\end{array}$ & $\begin{array}{l}\text { Ethereal } 11.63 \\
\text { Nemorino } 5.24\end{array}$ & 66.49 & Ethereal, 5-1: $11=1=1$ & 6 & 83.33 & '+' \\
\hline $\begin{array}{l}\text { Ko } \\
\text { Wa }\end{array}$ & 05 & $\begin{array}{l}\checkmark \\
\checkmark\end{array}$ & 250 & $\begin{array}{l}3824 \\
3574\end{array}$ & $\begin{array}{l}\mathrm{P} \\
\mathrm{Q}\end{array}$ & $\begin{array}{l}04 \\
29\end{array}$ & $\begin{array}{c}\text { Komodo } 2418.00 \\
\text { Wasp } 3.78\end{array}$ & 80.83 & Komodo, $41 \frac{1}{2-1 / 2}: 1=111$ & 5 & 90.00 & + \\
\hline $\begin{array}{l}\text { An } \\
\mathrm{Pe}\end{array}$ & 06 & $\begin{array}{l}x \\
x\end{array}$ & 72 & $\begin{array}{l}3683 \\
3611\end{array}$ & $\begin{array}{l}1 \\
2\end{array}$ & $\begin{array}{l}13 \\
20\end{array}$ & $\begin{array}{c}\text { Andscacs } 0.95123 \\
\text { Pedone } 230719\end{array}$ & 60.22 & Andscacs, $41 \frac{1}{2}-1 \frac{1}{2} 2$ : $11===1$ & 6 & 75.00 & + \\
\hline $\begin{array}{l}\mathrm{Sv} \\
\mathrm{Ru}\end{array}$ & 07 & $\begin{array}{l}\checkmark \\
\checkmark\end{array}$ & 235 & $\begin{array}{l}3785 \\
3550\end{array}$ & $\begin{array}{l}\mathrm{P} \\
2\end{array}$ & $\begin{array}{l}05 \\
28\end{array}$ & $\begin{array}{c}\text { StoofVlees II a12 } \\
\text { RubiChess } 1.6^{*}\end{array}$ & 79.46 & Stoofvlees, $41 \frac{1}{2}-2 \frac{1}{2} 2$ : $01=1=1=$ & 7 & 64.29 & - \\
\hline $\begin{array}{l}\mathrm{Xi} \\
\mathrm{Fi}\end{array}$ & 08 & $\begin{array}{l}\checkmark \\
\checkmark\end{array}$ & 6 & $\begin{array}{l}3732 \\
3726\end{array}$ & $\begin{array}{l}1 \\
2\end{array}$ & $\begin{array}{l}12 \\
21\end{array}$ & $\begin{array}{l}\text { Xiphos } 0.5 .6 \\
\text { Fire } 021819\end{array}$ & 50.86 & Xiphos, 41/2-31/2: $1=======$ & 8 & 56.25 & + \\
\hline $\begin{array}{l}\mathrm{St} \\
\mathrm{c} 22\end{array}$ & 09 & $\begin{array}{l}\checkmark \\
\checkmark\end{array}$ & 433 & $\begin{array}{l}3896 \\
3463\end{array}$ & $\begin{array}{l}\mathrm{P} \\
\mathrm{Q}\end{array}$ & $\begin{array}{l}02 \\
31\end{array}$ & $\begin{array}{l}\text { Stockfish } 19100908 \\
\text { chess } 22 \mathrm{k} 1.13\end{array}$ & 93.52 & Stockfish, 5-0: 11111 & 5 & 100.00 & + \\
\hline $\begin{array}{l}\mathrm{Gi} \\
\mathrm{rf}\end{array}$ & 10 & $\begin{array}{l}\checkmark \\
\checkmark\end{array}$ & 26 & $\begin{array}{l}3705 \\
3679\end{array}$ & $\begin{array}{l}1 \\
1\end{array}$ & $\begin{array}{l}15 \\
18\end{array}$ & $\begin{array}{c}\text { Ginkgo S13 } \\
\text { rofChade 2.204 }\end{array}$ & 46.38 & rofChade, $4 \frac{1}{2} 2-2^{1} \frac{1}{2}:===11==$ & 7 & 64.29 & + \\
\hline $\begin{array}{l}\mathrm{Km} \\
\mathrm{Va}\end{array}$ & 11 & $\begin{array}{l}\checkmark \\
\times\end{array}$ & 169 & $\begin{array}{l}3783 \\
3614\end{array}$ & $\begin{array}{l}1 \\
2\end{array}$ & $\begin{array}{l}07 \\
26\end{array}$ & $\begin{array}{c}\text { KomodoMCTS } 2418.00 \\
\text { Vajolet } 22.7\end{array}$ & 72.29 & KomodoMCTS, 5-2:==1==11 & 7 & 71.43 & $=$ \\
\hline $\begin{array}{l}\mathrm{La} \\
\mathrm{Fr}\end{array}$ & 12 & $\begin{array}{l}x \\
x\end{array}$ & 107 & $\begin{array}{l}3718 \\
3611\end{array}$ & $\begin{array}{l}1 \\
2\end{array}$ & $\begin{array}{l}10 \\
23\end{array}$ & $\begin{array}{c}\text { Laser } 230319 \\
\text { Fritz } 16.10\end{array}$ & 64.60 & Laser, 41/2-1/2: $11=11$ & 5 & 90.00 & +\# \\
\hline $\begin{array}{l}\mathrm{AS} \\
\mathrm{Ni}\end{array}$ & 13 & $\begin{array}{l}\checkmark \\
\times\end{array}$ & 347 & $\begin{array}{l}3832 \\
3485\end{array}$ & $\begin{array}{l}\mathrm{P} \\
\mathrm{Q}\end{array}$ & $\begin{array}{l}03 \\
30\end{array}$ & $\begin{array}{c}\text { AllieStein v0.5_c328142-n11 } \\
\text { Nirvana } 2.4\end{array}$ & 88.77 & AllieStein, 5-0: 11111 & 5 & 100.00 & + \\
\hline $\begin{array}{l}\mathrm{Fz} \\
\mathrm{Ar}\end{array}$ & 14 & $\begin{array}{l}x \\
\checkmark\end{array}$ & 22 & $\begin{array}{l}3659 \\
3637\end{array}$ & $\begin{array}{l}1 \\
2\end{array}$ & $\begin{array}{l}14 \\
19\end{array}$ & $\begin{array}{c}\text { Fizbo } 2 \\
\text { Arasan TCEC_CUP4 }\end{array}$ & 53.07 & Arasan, $41 / 2-21 / 2:===11==$ & 7 & 64.29 & + \\
\hline $\begin{array}{l}\mathrm{Ho} \\
\mathrm{Gu}\end{array}$ & 15 & $\begin{array}{l}x \\
x\end{array}$ & 198 & $\begin{array}{l}3798 \\
3600\end{array}$ & $\begin{array}{l}\mathrm{P} \\
2\end{array}$ & $\begin{array}{l}06 \\
27\end{array}$ & $\begin{array}{c}\text { Houdini } 6.03 \\
\text { Gull } 3\end{array}$ & 75.58 & Houdini, 5-0: 11111 & 5 & 100.00 & + \\
\hline $\begin{array}{l}\text { Ch } \\
\text { Bo }\end{array}$ & 16 & $\begin{array}{l}x \\
x\end{array}$ & -70 & $\begin{array}{l}3616 \\
3686\end{array}$ & $\begin{array}{l}1 \\
2\end{array}$ & $\begin{array}{l}11 \\
22\end{array}$ & $\begin{array}{c}\text { Chiron TCEC16 } \\
\text { Booot } 6.3 .1\end{array}$ & 40.32 & Chiron, $41 \frac{1}{2}-31 \frac{1}{2}$ : $====1===$ & 8 & 56.25 & + \\
\hline
\end{tabular}

LEELA CHESS ZERO, as the holder of the TCEC Cup, opened proceedings but was held to a draw four times by PIRARUCU. The adjacent seeds CHESSBRAINVB and JONNY took us into overtime after three wins on each side. CHESSBRAINVB was two down but came back with two wins as Black. One was to become the longest decisive game of the event at 201 moves with mate some nine moves away. SCORPIONN and ALLIESTEIN displayed none of LCZERO's reticence with unexpected 5-0 wins. ETHEREAL and STOCKFISH also achieved the whitewash, the latter greatly assisted by the fact that NEMORINO crashed three times on move 2 when playing White.

XIPHOS nosed ahead of FIRE and then, like Horatius on the bridge, refused to be passed: FIRE went out, 41/2-31/2. GINKGO and ROFCHADE were closely seeded so it was only a small surprise when this match provided the first win by the underdog. ARASAN in a new version then beat FIZBO, five seeds higher. With the crowd on their side, how far could these two victors go?

\footnotetext{
${ }^{2}$ In these tables, the first-named engine played White first except where indicated by a '*'. Alongside the ' $\% \mathrm{P}^{\prime}$ column, '+' ('-') indicates an unexpected excess (shortfall) of a $1 / 2$-point in the winner's score. ' $\# \mathrm{~g}$ ' $\equiv$ number of games.
} 
Table 2. TCEC Cup 4: round two results from the winner's perspective.

\begin{tabular}{|c|c|c|c|c|c|c|c|c|c|c|c|c|}
\hline$\alpha \beta$ & $\#$ & 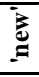 & $\begin{array}{c}\text { Elo } \\
\Delta \\
\end{array}$ & Elo & 尺્o & 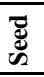 & Round 2 Pairings & $\mathbf{E} \% \mathbf{P}$ & Round 2 Results & $\# \mathbf{g}$ & $\% \mathbf{P}$ & par.. \\
\hline $\begin{array}{l}\mathrm{Lc} \\
\mathrm{Cb}\end{array}$ & 01 & $\begin{array}{l}\checkmark \\
\times\end{array}$ & 241 & $\begin{array}{l}3901 \\
3660\end{array}$ & $\begin{array}{l}\mathrm{P} \\
1\end{array}$ & $\begin{array}{l}01 \\
16\end{array}$ & $\begin{array}{c}\text { LCZero v0.22.0-nT2 } \\
\text { ChessBrainVB } 3.74\end{array}$ & 80.05 & Leela Chess Zero, 41/2-11/2:==111= & 6 & 75.00 & - \\
\hline $\begin{array}{l}\mathrm{Sn} \\
\mathrm{Et}\end{array}$ & 02 & $\begin{array}{l}\checkmark \\
\checkmark\end{array}$ & -39 & $\begin{array}{l}3707 \\
3746\end{array}$ & $\begin{array}{l}1 \\
1\end{array}$ & $\begin{array}{l}08 \\
09\end{array}$ & $\begin{array}{c}\text { ScorpioNN v3.0.3-n_maddex_INT8 } \\
\text { Ethereal } 11.63\end{array}$ & 44.57 & ScorpioNN, 51/2-41/2: ===10===; $1=$ & 10 & 55.00 & + \\
\hline $\begin{array}{l}\text { Ko } \\
\text { An }\end{array}$ & 03 & $\begin{array}{l}\checkmark \\
\times\end{array}$ & 141 & $\begin{array}{l}3824 \\
3683\end{array}$ & $\begin{array}{l}\mathrm{P} \\
1\end{array}$ & $\begin{array}{l}04 \\
13\end{array}$ & $\begin{array}{c}\text { Komodo } 2418.00 \\
\text { Andscacs } 0.95123\end{array}$ & 68.92 & Komodo, 5-1: 11==11 & 6 & 83.33 & + \\
\hline $\begin{array}{l}\mathrm{Sv} \\
\mathrm{Xi}\end{array}$ & 04 & $\begin{array}{l}\checkmark \\
\checkmark\end{array}$ & 53 & $\begin{array}{l}3785 \\
3732\end{array}$ & $\begin{array}{l}\mathrm{P} \\
1\end{array}$ & $\begin{array}{l}05 \\
12\end{array}$ & $\begin{array}{l}\text { StoofVlees II a12 } \\
\text { Xiphos 0.5.6 }\end{array}$ & 42.64 & Xiphos, $41 \frac{1}{2}-2 \frac{1}{2}$ : $==1==1=$ & 7 & 64.29 & +\# \\
\hline $\begin{array}{l}\text { St } \\
\text { ro }\end{array}$ & 05 & $\begin{array}{l}\checkmark \\
\checkmark\end{array}$ & 217 & $\begin{array}{l}3896 \\
3679\end{array}$ & $\begin{array}{l}\mathrm{P} \\
1\end{array}$ & $\begin{array}{l}02 \\
18\end{array}$ & $\begin{array}{l}\text { Stockfish } 19100908 \\
\text { rofChade } 2.204\end{array}$ & 77.62 & Stockfish, 5-1: $1=1=11$ & 6 & 83.33 & + \\
\hline $\begin{array}{l}\mathrm{Km} \\
\mathrm{La}\end{array}$ & 06 & $\begin{array}{l}\checkmark \\
\times\end{array}$ & 65 & $\begin{array}{l}3783 \\
3718\end{array}$ & $\begin{array}{l}1 \\
1\end{array}$ & $\begin{array}{l}07 \\
10\end{array}$ & $\begin{array}{c}\text { KomodoMCTS } 2418.00 \\
\text { Laser } 230319\end{array}$ & 59.00 & 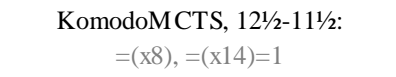 & 24 & 52.08 & --- \\
\hline $\begin{array}{l}\mathrm{AS} \\
\mathrm{Ar}\end{array}$ & 07 & $\begin{array}{l}\checkmark \\
\checkmark\end{array}$ & 195 & $\begin{array}{l}3832 \\
3637\end{array}$ & $\begin{array}{l}\mathrm{P} \\
2\end{array}$ & $\begin{array}{l}03 \\
19\end{array}$ & $\begin{array}{c}\text { AllieStein v0.5_c328142-n11 } \\
\text { Arasan TCEC_CUP4 }\end{array}$ & 75.25 & AllieStein, 41/2-11/2: ==111= & 6 & 75.00 & $=$ \\
\hline $\begin{array}{l}\mathrm{Ho} \\
\mathrm{Ch}\end{array}$ & 08 & $\stackrel{x}{r}$ & 182 & $\begin{array}{l}3798 \\
3616\end{array}$ & $\begin{array}{l}\mathrm{P} \\
1\end{array}$ & $\begin{array}{l}06 \\
11\end{array}$ & $\begin{array}{c}\text { Houdini } 6.03 \\
\text { Chiron TCEC16 }\end{array}$ & 73.79 & Houdini, $41 / 2-1 \frac{1}{2} 2:=1=11=$ & 6 & 75.00 & $=$ \\
\hline
\end{tabular}

LEELA CHESS ZERO continued to see off the lowest remaining seed respectfully, this time more convincingly if still a touch under par. SCORPIONN-ETHEREAL and KOMODO-ANDSCACS had to be rerun after longtime spectator 'Occyroexanthub' noticed the latter engine was running over $40 \%$ slower than expected. ${ }^{3}$ It was discovered that CHESSBRAINVB had not entirely left the field of play: kudos to 'Aloril' for managing the contingency so promptly. The match results were unchanged even though the game results were. The much improved XIPHOS took the first major scalp, beating new Premier Division addition STOOFVLEES.

In the lower half, STOCKFISH made short work of ROFCHADE, unswayed by the partial crowd and the latter's garlands as underdog winner. In complete contrast, KOMODOMCTS and LASER then contrived a record-length sequence of 23 draws before KOMODOMCTS won as Black. Their ELO ratings suggested that only eight games would be required. ALLIESTEIN and HOUDINI came through as expected, $4 \frac{1}{2}-1 \frac{1}{2}$, against ARASAN and CHIRON respectively.

\section{The quarter-finals, semi-finals, third-place play-off and final}

And so we moved, Wimbledon style, into the second week with its higher ticket prices and even more expensive strawberries. The top eight took their places for the quarterfinals with the exception of STOOFVLEES whose early demise had benefitted XIPHOS, seed \#12. LEELA was untroubled by SCORPIONN, remained unbeaten so far and was basically winning three points from four. KOMODO had a stiffer challenge from XIPHOS but was also unbeaten. KOMODOMCTS could not lay a hand on STOCKFISH so we were denied a battle of the dragons this time. At the last moment, HouDINI levelled at $4-4$, courtesy of the inexplicable 19...Bxa3 from ALLIESTEIN. After ten more draws, ALLIESTEIN won as Black to close the match.

\footnotetext{
3 'ChessBrainVB', as $20^{+}$processes each taking $2.3 \%$ of the CPU, was the ghost in the machine. The SCORPIONN-

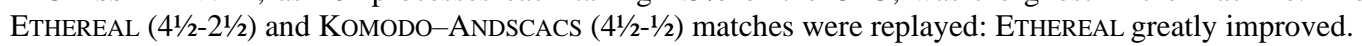


Table 3. TCEC Cup 4: quarterfinal results from the winner's perspective.

\begin{tabular}{|c|c|c|c|c|c|c|c|c|c|c|c|}
\hline$\alpha \beta$ & $\#$ & 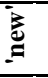 & $\begin{array}{c}\text { Elo } \\
\Delta\end{array}$ & Elo & 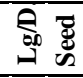 & Quarter-final Pairings & E\%P & Quarter-final Results & $\# \mathbf{g}$ & $\% \mathbf{P}$ & par.. \\
\hline $\begin{array}{l}\mathrm{Lc} \\
\mathrm{Sn}\end{array}$ & 01 & $\begin{array}{l}\checkmark \\
\checkmark\end{array}$ & 194 & $\begin{array}{l}3901 \\
3707\end{array}$ & $\begin{array}{ll}P & 01 \\
1 & 08\end{array}$ & $\begin{array}{c}\text { LCZero v0.22.0-nT2 } \\
\text { ScorpioNN v3.0.3-n_maddex_INT8 }\end{array}$ & 75.14 & LCZero, $41 / 2-1 \frac{1}{2} 2: 1=11==$ & 6 & 75.00 & $=$ \\
\hline $\begin{array}{l}\text { Ko } \\
\text { Xi }\end{array}$ & 02 & $\begin{array}{l}\checkmark \\
\checkmark\end{array}$ & 92 & $\begin{array}{l}3824 \\
3732\end{array}$ & $\begin{array}{ll}P & 04 \\
1 & 12\end{array}$ & $\begin{array}{l}\text { Komodo } 2418.00 \\
\text { Xiphos 0.5.6 }\end{array}$ & 62.63 & Komodo, 5-2: 1===1=1 & 7 & 71.43 & + \\
\hline $\begin{array}{l}\mathrm{St} \\
\mathrm{Km}\end{array}$ & 03 & $\checkmark$ & 113 & $\begin{array}{l}3896 \\
3783\end{array}$ & $\begin{array}{ll}P & 02 \\
1 & 07\end{array}$ & $\begin{array}{c}\text { Stockfish } 19100908 \\
\text { KomodoMCTS } 2418.00\end{array}$ & 65.38 & Stockfish, $4 \frac{1}{2}-1 \frac{1}{2}: 1=1=1=$ & 6 & 75.00 & + \\
\hline $\begin{array}{l}\text { AS } \\
\text { Ho }\end{array}$ & 04 & $\checkmark$ & 34 & $\begin{array}{l}3832 \\
3798\end{array}$ & $\begin{array}{ll}\mathrm{P} & 03 \\
\mathrm{P} & 06\end{array}$ & $\begin{array}{c}\text { AllieStein v0.5_c328142-n11 } \\
\text { Houdini } 6.03\end{array}$ & 54.74 & $\begin{array}{l}\text { AllieStein, } 10^{1 / 2-9} \frac{1}{2} \text { : } \\
===1===0,=(x 10)=1\end{array}$ & 20 & 52.50 & - \\
\hline
\end{tabular}

Rugby World Cup semi-finals in Japan, TCEC semi-finals in cloud-land: what's not to like! The 'last four' saw all the top seeds in place for the first time. Indeed, results went with the seeding and with only two tiebreaker games although the Elo figures were close. The $4^{\text {th }}$ TCEC Cup therefore brought about the final that many were expecting in the main TCEC16 event, a match between LEELA CHESS ZERO, the Cup holder, and the Lewis Hamilton of TCEC, STOCKFISH.

Table 4. TCEC Cup 4: semi-final results from the winner's perspective.

\begin{tabular}{|c|c|c|c|c|c|c|c|c|c|c|c|}
\hline$\alpha \beta$ & $\#$ & $\mathrm{E}$ & $\begin{array}{c}\text { Elo } \\
\Delta\end{array}$ & Elo & 毞 总 & Semi-final Pairings & E\%P & Semi-final Results & $\# \mathbf{g}$ & $\% \mathbf{P}$ & par.. \\
\hline $\begin{array}{l}\text { Lc } \\
\text { Ko }\end{array}$ & 01 & $\begin{array}{l}2 \\
\checkmark\end{array}$ & 77 & $\begin{array}{l}3901 \\
3824\end{array}$ & \begin{tabular}{|ll}
$\mathrm{P}$ & 01 \\
$+\mathrm{P}$ & 04
\end{tabular} & $\begin{array}{c}\text { LCZero v0.22.0-nT2 } \\
\text { Komodo } 2418.00\end{array}$ & \} 60.62 & LCZero, $4 \frac{1}{2}-3-3 \frac{1}{2}:====101=$ & 8 & 56.25 & - \\
\hline $\begin{array}{c}\text { St } \\
\text { AS }\end{array}$ & 02 & $\checkmark$ & 64 & $\begin{array}{l}3896 \\
3832\end{array}$ & $\begin{array}{ll}\mathrm{P} & 02 \\
\mathrm{P} & 03\end{array}$ & $\begin{array}{c}\text { Stockfish } 19100908 \\
\text { AllieStein v0.5_c328142-n11 }\end{array}$ & 58.86 & Stockfish, $5^{1 / 2-4}$ & 10 & 55.00 & \\
\hline
\end{tabular}

But first, the play-off for the bronze medal. ALLIESTEIN took the sole win in game 2, see Figs. 2a-b. ALLIESTEIN's confidence increased around position 23w: it seemed to see things before kibitzing REDFISH. KOMODO saw danger only around move 51.
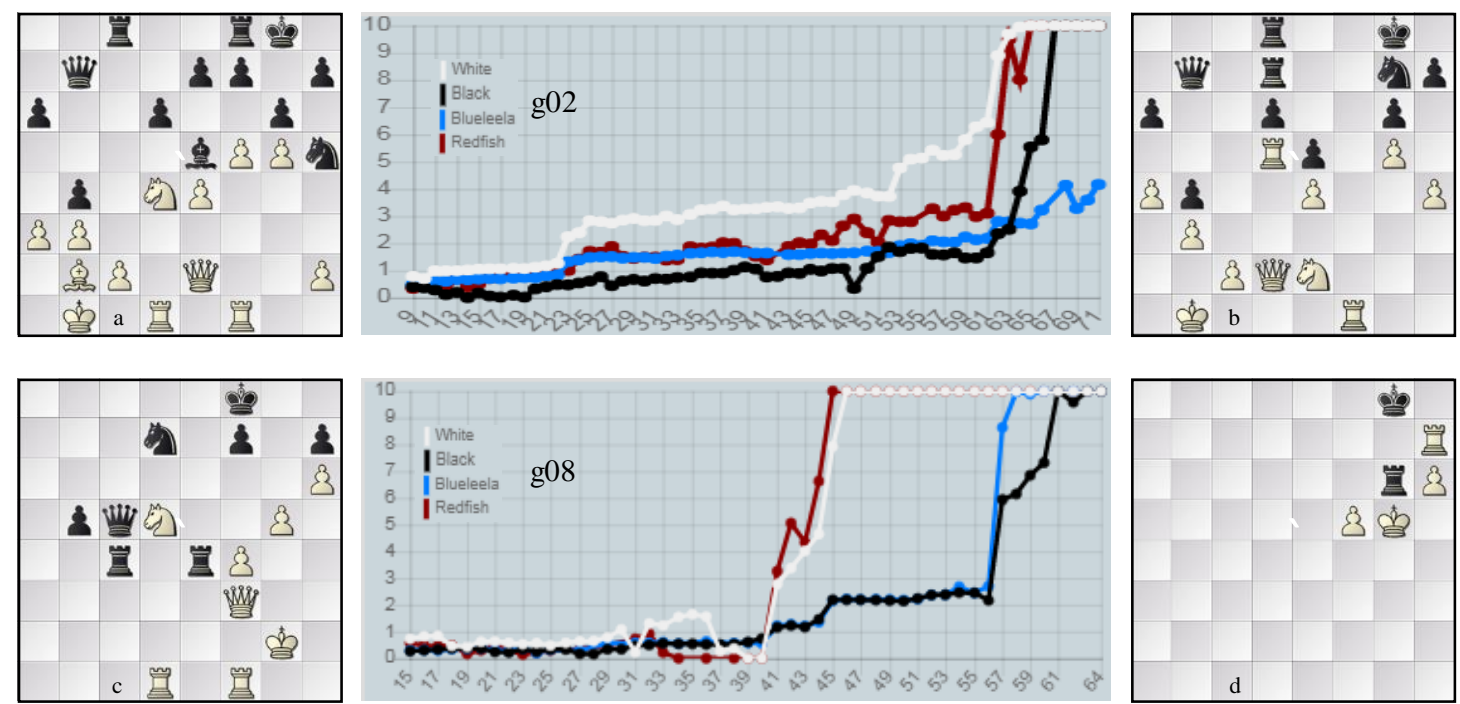

Fig. 2.(a) Bronze match, ALLIESTEIN-Komodo game 2: position 23w, evaluation graph and (b) position 51w; (c) the Cup Final, STOCKFISH-LEELA game 8: position 40b, the evaluation-graph, and (d) White on $65^{\text {th }}$ move. 
The final somewhat surprisingly ran to only eight games, with STOCKFISH denying LEELA CHESS ZERO the chance of reply by winning the last. The position seemed drawn at move 40b, Fig. 2c, although it is possible that STOCKFISH had accumulated advantages which would only materialise over its horizon. STOCKFISH and other Shannon-AB engines recommended 40...Rcd4 so 40...Qc6 must have come as a pleasant surprise. White's evaluation shot up but LEELA remained unaware until move 56 . The closing position, Fig. 2d, is hardly an exercise for the reader: mate in four moves.

Table 5. TCEC Cup 4: the Komodo - AllieStein play-off and the LeEla CHESS ZeRO - StockfiSh final.

\begin{tabular}{|c|c|c|c|c|c|c|c|c|c|c|c|}
\hline$\alpha \beta$ & $\#$ & $\stackrel{3}{\stackrel{3}{g}}$ & $\begin{array}{c}\text { Elo } \\
\Delta\end{array}$ & Elo & 实 & Small Final \& Final Pairings & $\mathbf{E} \% \mathbf{P}$ & Results & $\# \mathbf{g}$ & $\% \mathbf{P}$ & par.. \\
\hline $\begin{array}{l}\text { AS } \\
\text { Ko }\end{array}$ & 02 & $\begin{array}{l}\checkmark \\
\checkmark\end{array}$ & 8 & $\begin{array}{l}3832 \\
3824\end{array}$ & $\begin{array}{ll}\mathrm{P} & 03 \\
\mathrm{P} & 04\end{array}$ & $\begin{array}{c}\text { AllieStein v0.5_c328142-n11 } \\
\text { Komodo } 2418.00^{*}\end{array}$ & 51.12 & AllieStein, 41/2-31/2: =1====== & 8 & 56.25 & + \\
\hline $\begin{array}{l}\mathrm{Lc} \\
\mathrm{St}\end{array}$ & 01 & $\begin{array}{l}\checkmark \\
\checkmark\end{array}$ & 5 & $\begin{array}{l}3901 \\
3896\end{array}$ & $\begin{array}{ll}\mathrm{P} & 01 \\
\mathrm{P} & 02\end{array}$ & $\begin{array}{c}\text { LCZero v0.22.0-nT2 } \\
\text { Stockfish } 19100908\end{array}$ & 49.30 & Stockfish, 41/2-31/2: =======1 & 8 & 56.25 & + \\
\hline
\end{tabular}

\section{$4 \quad$ In conclusion}

Congratulations to STOCKFISH, the other medallists and all participants. The last game of the TCEC Cup final closes another successful TCEC season. We continue to see the rise of the 'new generation' neural-network and/or MCTS engines and these bring new styles of play to the board. For the first time, we have two not unrelated NN-engines on the podium with one resilient Shannon representative, STOCKFISH, holding out for us old-timers.

All the games are available (Haworth and Hernandez, 2019b) in pgn files, some with example play outs: an excess of riches. Table 6 highlights the longest and shortest games. We may all enjoy these high tempo but super-GM games at our leisure while anticipating TCEC 17. Until next time!

Table 6. The shortest and longest 1-0, drawn and 0-1 games in each phase of TCEC Cup 4:

' $40 / 1$ ' in row 1, column 2 for example means 'game 40 in the pgn, game 1 in the match'.

\begin{tabular}{|c|c|c|c|c|c|c|c|c|c|c|c|c|c|c|c|c|c|c|}
\hline \multirow{3}{*}{ Round } & \multicolumn{6}{|c|}{$1-0$} & \multicolumn{6}{|c|}{$1 / 2-1 / 2$} & \multicolumn{6}{|c|}{$0-1$} \\
\hline & \multicolumn{3}{|c|}{ Shortest } & \multicolumn{3}{|c|}{ Longest } & \multicolumn{3}{|c|}{ Shortest } & \multicolumn{3}{|c|}{ Longest } & \multicolumn{3}{|c|}{ Shortest } & \multicolumn{3}{|c|}{ Longest } \\
\hline & \multicolumn{2}{|c|}{ Game } & \multirow{2}{*}{ \#mv } & \multicolumn{2}{|c|}{ Game } & \multirow{2}{*}{$\begin{array}{r}\text { \#mv } \\
145\end{array}$} & \multicolumn{2}{|c|}{ Game } & \multirow{2}{*}{$\begin{array}{c}\# \mathbf{m v} \\
25\end{array}$} & \multicolumn{2}{|c|}{ Game } & \multirow{2}{*}{$\begin{array}{r}\# \mathbf{m v} \\
161\end{array}$} & \multicolumn{2}{|c|}{ Game } & \multirow[t]{2}{*}{$\# \mathbf{m v}$} & \multicolumn{2}{|c|}{ Game } & \multirow{2}{*}{$\begin{array}{r}\# \mathbf{m v} \\
201\end{array}$} \\
\hline 1 & $40 / 1$ & $\mathrm{Ru}-\mathrm{Sv}$ & & $22 / 5$ & Sn-Te & & $68 / 2$ & Va-Km & & $6 / 6$ & Pi-Lc & & $56 / 2$ & c22-St & & $13 / 6$ & Jo-Cb & \\
\hline 2 & $5 / 5$ & $\mathrm{Lc}-\mathrm{Cb}$ & 49 & $64 / 5$ & AS-Ar & 99 & $2 / 2$ & $\mathrm{Cb}-\mathrm{Lc}$ & 23 & $65 / 6$ & Ar-AS & 201 & $25 / 3$ & $\mathrm{~Sv}-\mathrm{Xi}$ & 51 & $11 / 5$ & Sn-Et & 94 \\
\hline QF & $27 / 8$ & Ho-AS & 33 & $14 / 1$ & St-Km & 98 & $19 / 6$ & $\mathrm{Km}-\mathrm{St}$ & 35 & $28 / 9$ & AS-Ho & 343 & $39 / 20$ & Ho-AS & 83 & $23 / 4$ & Ho-AS & 103 \\
\hline SF & $6 / 6$ & Ko-Lc & 54 & $7 / 7$ & Lc-Ko & 69 & $2 / 2$ & Ko-Lc & 58 & $1 / 1$ & Lc-Ko & 121 & - & - & - & - & - & - \\
\hline for 3rd & $2 / 2$ & AS-Ko & 71 & $2 / 2$ & AS-Ko & 71 & $8 / 8$ & AS-Ko & 31 & $6 / 6$ & AS-Ko & 200 & - & - & - & - & - & - \\
\hline Final & $8 / 8$ & St-Lc & 64 & $8 / 8$ & St-Lc & 64 & $6 / 6$ & St-Lc & 33 & $3 / 3$ & Lc-St & 105 & - & - & - & - & - & - \\
\hline Overall & 1,40 & $\mathrm{Ru}-\mathrm{Sv}$ & 31 & 1,22 & Sn-Te & 145 & 2,2 & $\mathrm{Cb}-\mathrm{Lc}$ & 23 & QF, 28 & AS-Ho & 343 & 1,56 & c22-St & 48 & 1,13 & Jo- $\mathrm{Cb}$ & 201 \\
\hline
\end{tabular}

\section{REFERENCES}

CPW (2019) https://tinyurl.com/icga046. Biographies of chess engines, authors and developers.

Haworth, G. $M^{\mathrm{c}}$ C. and Hernandez, N. (2019a). http://centaur.reading.ac.uk/83156/. TCEC16: the $16^{\text {th }}$ Top Chess Engine Championship. ICGA Journal 41(4), 241-252.

Haworth, G. Mc C. and Hernandez, N. (2019b). http://centaur.reading.ac.uk/86831/. TCEC Cup 4. This note plus annotated statistics and pgn files. ICGA Journal 41(4), 259-263. 